\title{
Lock-n-Sync for Secure Data Storage in Shared Electron Microscopy Facilities
}

\author{
Kuilin Li and Shuyou Li
}

FOM Networks, Inc. One Northfield Plaza, Suite 300, Northfield, IL 60093-1214.

Over the past two decades, nearly all electron microscopy facilities have finished the transition from analog imaging to digital imaging. Instead of working in the darkroom and struggling with stacks and stacks of negative films, more and more of our data is in the form of digital micrographs stored on our computers. While enjoying the convenience of digital imaging, we are facing new challenges of data storage and data security. In fear of computer viruses and internet hackers, a lot of EM managers have disconnected their instrument-control PCs from the internet. Since the USB flash drive is getting more and more popular, they have sealed USB ports to prevent flash drives from spreading viruses. In some laboratories, EM users are often forced to use CD or DVD discs to retrieve data from the lab after the experiments. Some other labs have come to the next level and implemented a local area network (LAN) to store EM data on a local file server.

For the convenience of data retrieval and data backup, the experimental data, in most shared EM facilities, is transparent to all the facility users. Any user can peek or make copies of another user's data at any time. Some cautious users would remove their data from the shared file server as soon as the data is copied to their personal devices, then they lost the centralized back up of raw experimental data. This is apparently against the rule of thumb in data security. In the FDA's Code of Federal Regulations on Electronic Records (CFR 21 part 11), for Electronic Records, “...Such procedures and controls shall include... protection of records to enable their accurate and ready retrieval throughout the records retention period and limiting system access to authorized individuals..." [1].

In this work we demonstrated our best of practices to store, transmit, and secure the large data sets in a shared facility. Our procedures include a seamless integration of the FOMC online calendar with an access control software called Lock-n-Sync [2]. With this system, only authorized users are able to reserve time and use the microscope; only the user who booked time on the microscope may operate the instrument; and the operator may only view and store data in his/her own designated space. All user data collected during operation is automatically synchronized and backed up under the specific user's account on a shared server. The Lock-n-Sync software may also be used with any institutional level single-sign-on protocol, such as Active Directory, CAS, SAML, Shibboleth, and so on.

A typical use case of the FOM® online calendar and the Lock-n-Sync software is depicted in Figure 1, which includes

Step (a). A user reserves instrument time with the FOM ${ }^{\circledR}$ online calendar, typically one week before the experiment time. The instrument manager has the right to grant user's access levels, define reservation rules, and billing policies where applicable.

Step (b). Before using the reserved instrument, a user must login to Lock-n-Sync system, which is installed on the instrument-control computer. The Lock-n-Sync system then authenticates the user using the FOM central database. 
Step (c). During the experiment, the Lock-n-Sync software monitors all the data that this user collects on the microscope and automatically synchronizes it with the remote file server under this user's profile.

Step (d). After the experiment, a user may access the data stored on the central data storage via the FOM ${ }^{\circledR}$ file browser. By default a user may only browse their own data. A user may also appoint another $\mathrm{FOM}{ }^{\circledR}$ user as a collaborator and grant them access to some of their data.

References:

[1] FDA Code of Federal Regulations, Electronic Records; Electronic Signatures, 21 C.F.R. § 11.10 (2014), available at

http://www.accessdata.fda.gov/scripts/cdrh/cfdocs/cfCFR/CFRSearch.cfm?CFRPart=11\&showFR=1

[2] Lock-n-Sync is a patent-pending software by FOM Networks, Inc. For more information please contact Dr. Shuyou Li at FOM Networks, Inc.

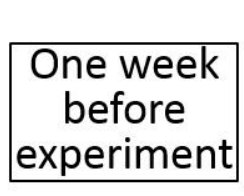

(a)

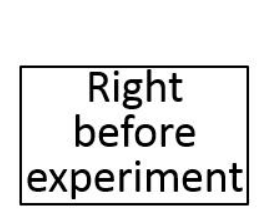

(b)

\begin{tabular}{c|c|}
\hline During \\
experiment
\end{tabular}

(c)

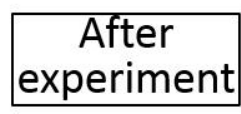

(d)

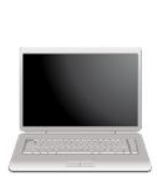

User

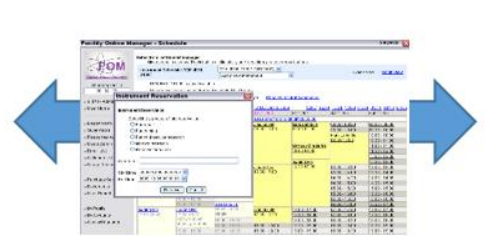

Reserve time on FOM Calendar

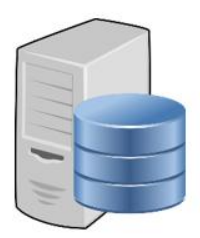

FOM Server

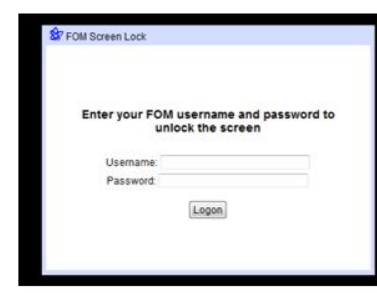

FOM Screen Lock

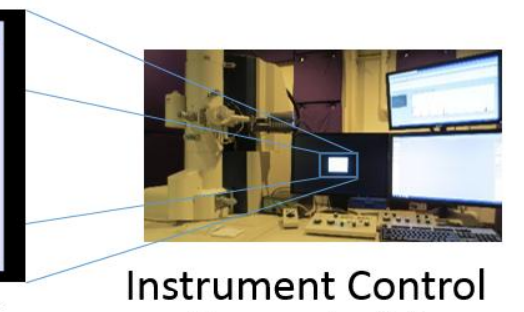

Computer(s)

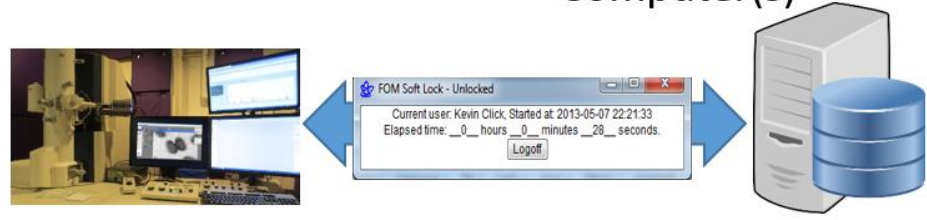

Data Acquisition FOM Lock-n-Sync Data Storage

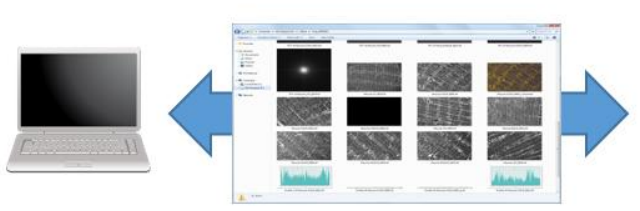

User File Browser with FOM Authentication

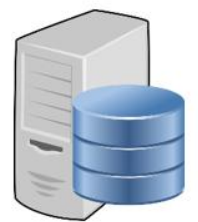

Data Storage

Figure 1. A typical use case of FOM ${ }^{\circledR}$ calendar and Lock-n-Sync system in an electron microscopy laboratory. 